\title{
Attention Bias to and Recognition of Sexual Images
}

1 *Ondřej Novák, MA ${ }^{1,4 *}$, Klára Bártová, $\mathrm{PhD}^{1,2}$, Václav Vagenknecht, $\mathrm{MA}^{\mathbf{1}}, \boldsymbol{\&}$ Kateřina

2 Klapilová, $\mathbf{P h D}^{1,3}$

$3{ }^{1}$ Laboratory of Evolutionary Sexology and Psychopathology, National Institute of Mental Health,

4 Klecany, Czech Republic

$5 \quad{ }^{2}$ Institute of Sexology, First Faculty of Medicine, Charles University, Prague, Czech Republic

$6 \quad{ }^{3}$ Faculty of Humanities, Charles University, Prague, Czech Republic

$7 \quad{ }^{4}$ Department of Psychology, Faculty of Arts, Charles University, Prague, Czech Republic

8 * Correspondence:

9 Ondřej Novák, MA

10 Laboratory of Evolutionary Sexology and Psychopathology, National Institute of Mental Health,

11 Topolová 748, 25067 Klecany, Czech Republic

12 ondrej.novak@nudz.cz

13 Keywords: Attention, Visuospatial Attention, Sexual, Erotic, Dot probe, Recognition, Pictures, 14 Images.

15 Abstract

Attention to sexual stimuli is necessary for the development of sexual response, yet while there is some evidence of attention bias in favor of sexual stimuli, the direction and magnitude of the effect remain so far unknown. A high-powered sample of 113 participants was tested using the dot-probe task and picture recognition task to measure visuospatial attention to erotic images. Participants showed no attention bias in the dot-probe task $(r B=0.201, \mathrm{p}=0.064)$ but were significantly better at recognizing erotic rather than neutral or training pictures $(d=1.445$ and 1.461 , respectively, both $p<$ 0.001). These results indicate that spatial attention bias to sexual pictures is small, negligible, eventually nonexistent, or else the dot-probe task is not a reliable tool to assess it. Results of the picture recognition task, on the other hand, show that sexual stimuli are prioritized in memory and this should be explored in detail in future research.

\section{Introduction}

It is widely assumed that sexual stimuli capture attention. This is reflected in, for instance, the frequent use of sexual content in advertising (Hennink-Kaminski \& Reichert, 2011; Reichert \& Carpenter, 2003; Reichert, Lambiase, Morgan, Carstarphen, \& Zavoina, 1999). It has been demonstrated that attentional processes play a central role in sexual arousal (Barlow, 1986; de Jong, 2009) and it is believed that they are processed much like other evolutionarily meaningful stimuli (Spiering \& Everaerd, 2007). Nearly all theoretical concepts which operate with attention and sexual stimuli assume some form of attention bias. Surprisingly, though, only a handful of studies had so far investigated the particular patterns of attention toward sexual stimuli and even less studies employed cognitive tasks to do so. 
The dot-probe task (also known as serial probe task, attentional deployment task, or visual probe task) is - alongside the modified Stroop task, visual search task, and eye-tracking, neither of which are discussed here (for a review, see Jiang \& Vartanian, 2018) - one of the most widely used tool used to assess attention bias. Introduced in a context of research of attention to threat-related words in a clinically anxious population (MacLeod, Mathews, \& Tata, 1986), the dot-probe task was developed to measure early automatic allocation of spatial attention. It operates on a simple assumption that people respond faster to probes presented in an attended rather than unattended area of visual display (Navon \& Margalit, 1983; Posner, Snyder, \& Davidson, 1980). In a typical setup, a participant is presented with pairs of stimuli (in a mixed pair trial e.g. sexual and neutral pictures or words), which are then replaced by a probe that appears in the location of one of the stimuli. Participants are instructed to localize or identify the probe as quickly as possible. A faster response to a probe appearing in the location of the sexual stimulus would suggest that attention was directed toward the sexual stimulus (vigilance), while a slower response would indicate that attention was directed away from it (avoidance). The difference in mean response times between trials where a probe replaced a neutral picture and those where a probe replaced a sexual picture amounts to attention bias index, extreme scores of which are thought to reflect attention biases (MacLeod et al., 1986).

The main advantage of the dot-probe task over other methods of assessing attention bias is that it reflects a distinction between attentional vigilance and avoidance and its ecological validity can be increased by the use of pictures instead of words (Jiang \& Vartanian, 2018). Moreover, it can be used to examine the progress of attention by varying the duration of exposure of stimulus pairs. And while there is considerable variation in stimulus exposure time - it can vary from $100 \mathrm{~ms}$ (Cooper \& Langton, 2006) to 2,000 ms (Pottage \& Schaefer, 2012) - most studies present the stimuli for $500 \mathrm{~ms}$ (Kagerer et al., 2014; Mogg, Philippot, \& Bradley, 2004; Prause, Janssen, \& Hetrick, 2008).

60 The dot-probe task is used widely. It found its way into various research areas including eating disorders (Aspen, Darcy, \& Lock, 2013), chronic pain (Dehghani, Sharpe, \& Nicholas, 2003), smoking (Hogarth, Mogg, Bradley, Duka, \& Dickinson, 2003), alcohol (Townshend \& Duka, 2001), phobias (Wenzel \& Holt, 1999), anxiety (B. P. Bradley, Mogg, Falla, \& Hamilton, 1998), and others. In short, it is at present one of the most widely used methods for assessing attention bias in both clinical and non-clinical populations.

Although the importance of attention in sexual functioning is beyond doubt, since it determines whether a sexual response will occur (Barlow, 1986), there is so far no clear consensus on how exactly people attend to sexual stimuli. Recently, Strahler and colleagues (Strahler, Baranowski, Walter, Huebner, \& Stark, 2019) published a meta-analysis on attention bias towards and distractibility by sexual cues. Combining the results from 2,933 participants in 32 studies (including eight studies that used the dot-probe task) they found a medium-size effect of $g_{z}=0.49,95 \% C I$ $[0.37,0.61]$ for attention to sexual cues in general and an effect size of $g_{z}=0.34,95 \% C I[0.17,0.50]$ for the dot-probe task (and letter probe) in particular. However, due to differences in the methodologies used, the attention bias as measured by this meta-analysis combined several, and sometimes opposite, effects. To provide a more detailed analysis, we offer in the following a full review of studies which assessed attention bias to sexual pictures using the dot-probe task.

The first one to measure attention bias to sexual stimuli using a picture dot-probe task were Prause and colleagues (2008), who focused on the relationship between attentional and emotional response to sexual stimuli and participants' sexual desire. Although the authors did not report exact values, their results suggest that in mixed pair trials, participants displayed slower reactions to probes which 
replaced sexual pictures than to those where probes replaced neutral pictures. More generally, participants exhibited slower reactions in all trials containing sexual pictures than in trials that used pairs neutral stimuli $\left(\eta_{p}^{2}=0.46\right)$. This effect was even more pronounced in participants with higher levels of sexual desire and in trials with more explicit sexual content $\left(\eta^{2}{ }_{p}=0.12\right.$ and 0.31 , respectively). A similar pattern of response times was found in a study which compared attention bias for sexual pictures in women with and without the hypoactive sexual desire disorder (Brauer et al., 2012). As in the previous study, participants were in mixed pair trials slower at detecting probes which replaced sexual images compared to probes replacing neutral pictures. At the same time, their responses were fastest in trials containing neutral pairs. These results held for both the experimental and control group $\left(\eta^{2}=0.34\right)$. Given the fact that sexual stimuli are considered highly salient (Spiering \& Everaerd, 2007) and as such should attract attention, thus allowing for faster reactions to replacement by a probe, these results are somewhat counterintuitive. Prause and colleagues explained their findings in terms of delayed disengagement and inhibition to return (Posner \& Cohen, 1984), meaning that participants' attention was overwhelmed by the sexual content and could not disengage from it very fast. Such effect was observed in highly anxious individuals who found it difficult to disengage from fear-inducing but not sad or other emotional stimuli (Georgiou et al., 2005; Rinck, Becker, Kellermann, \& Roth, 2003). Some authors call this rather consistent effect 'sexual content-induced delay' (Imhoff, Barker, \& Schmidt, 2019).

Several later studies used a picture dot-probe task in slightly different contexts. Doornwaard and colleagues investigated how short erotic film clips affect the performance of the task (Doornwaard, van Den Eijnden, Johnson, \& ter Bogt, 2014). Once again, participants responded faster to neutral pair trials than to mixed pair trials containing sexual pictures $\left(\eta^{2}{ }^{2}=.22\right)$. In mixed pair trials, however, they responded faster to probes replacing sexual pictures than to probes replacing neutral pictures $\left(\eta^{2}{ }_{p}=0.07\right)$. Viewing of the erotic clips had no effect on their performance.

Kagerer and colleagues added a fourth type of trial, where both of the presented pictures were of a sexual nature (Kagerer et al., 2014). Similarly to the previous study, they found that participants reacted faster to probes replacing sexual pictures in mixed pair trials and to probes in neutral pair trials in general, and their reactions were in both cases faster than in mixed pair trials where the probe replaced the neutral picture $\left(\eta^{2}=0.027\right.$ and 0.018 , respectively). Nevertheless, their reactions in neutral pair trials in general did not differ from either sexual pair trials in general or mixed pair trials where the probe replaced a sexual picture.

Another study compared the attention bias index in individuals with and without compulsive sexual behavior (Mechelmans et al., 2014). Both the clinical and the control group had positive and nonzero attention bias towards explicit sexual stimuli, which in mixed pair trials manifested itself in faster responses to probes replacing sexual pictures than to those that replaced neutral pictures. Regrettably, the authors provided no values or effect sizes besides p-values. Employing the mTurk web platform, Seehuus (2015) compared different exposition times in the dot-probe task. In mixed pair trials, he found a significant attention bias toward probes following after sexual pictures for exposition times of $50 \mathrm{~ms}, 500 \mathrm{~ms}$, and $1250 \mathrm{~ms}\left(\eta^{2}=0.129,0.021\right.$ and 0.008 , respectively $)$. The study used no neutral pair trials. Following up with two experiments, Snowden and colleagues focused on gender-specific differences in the target stimuli (Snowden, Curl, Jobbins, Lavington, \& Gray, 2016). In the first experiment, rather than pairing a sexual picture with a neutral one, they used pictures containing a nude male and a nude female. Their study employed shorter exposition times (200 ms). Their results showed that heterosexual men reacted faster when probe replaced a female picture $\left(\eta^{2}=0.57\right)$, while in heterosexual women, no such difference was found. In a second experiment, they added neutral pictures. In line with the previous experiment, both men and women 
127 reacted faster to probes that followed female pictures in both male-female $\left(\eta^{2}=0.172\right.$ and 0.075 , 128 respectively) and female-neutral trial $\left(\eta^{2}=0.265\right.$ and 0.075 , respectively), while in male-neutral 129 trials, there was no difference in reaction times. So far, this is the only study that found any 130 difference between men and women using the dot-probe paradigm.

131 While most of these reviewed studies showed that the presence of sexual pictures influences the attention to probes, the magnitude and direction of the attention bias turned out to be highly inconsistent (MacLeod et al., 1986). Some studies that used mixed pair trials reported faster reactions to a probe replacing a sexual picture (Doornwaard et al., 2014; Kagerer et al., 2014; Mechelmans et al., 2014; Seehuus, 2015; Snowden et al., 2016), while others observed slower responses in such cases (Brauer et al., 2012; Prause et al., 2008).

Such mixed evidence may be the result of low reliability of the dot-probe paradigm as such (Schmukle, 2005; Staugaard, 2009). After all, an unreliable tool measures only error variance, thus leading to inconsistent results across studies (Schmukle, 2005). Snowden et al. (2016) suggest that the relatively low reliability of dot-probe task studies could be linked to a small size or perhaps even nonexistence of the effect they are trying to detect. Another source of this inconsistency could be publication bias. Publication bias is widespread in cognitive sciences in general (Ioannidis, Munafò, Fusar-Poli, Nosek, \& David, 2008) and in research on attention to sexual stimuli research in particular (Strahler et al., 2019). Complete absence of nonsignificant results in published studies seems to support this explanation. In conjunction with high heterogeneity in the reviewed studies (Strahler et al., 2019), it shows a clear need of more high-powered simple studies employing the dotprobe task. The aim of the present study is to contribute to our knowledge of the magnitude and direction of the assumed attention bias towards sexual stimuli in nonclinical population as measured by the dot-probe task.

150 Further, in the light of concerns about dot-probe task's ability to assess early automatic allocation of spatial attention (see e.g. Cooper \& Langton, 2006), it would be desirable to employ some validation measure (e.g. Miller \& Fillmore, 2010). One way to validate the results could be by a picture recognition task. Previous studies arrived at the three following observations: 1) subjects tend to spend more time looking at highly valued pictures, 2) they recognize pictures they looked at longer irrespectively of picture evaluation, and 3) subjects form no memory of pictures viewed only peripherally (Loftus, 1972). Based on the logic of the dot-probe task, MadLeod and colleagues (1986) suggested that sexual stimulus should capture participant's attention as soon as it appears and remain its focus it until a probe is revealed. Attention should thus be largely directed at sexual pictures. Since attention to a stimulus is crucial for a successful creation of memory of it (Bush \& Geer, 2001; Pottage \& Schaefer, 2012), we therefore predict that if the dot-probe task works as suggested, subjects should be able to recognize significantly more sexual than neutral pictures. In accordance with this reasoning, we employed in our study a simple picture recognition task. Previously, such recognition tasks were used with sexual stimuli only in the context of advertisement research (for a review, see Wirtz, Sparks, \& Zimbres, 2017). To the best of our knowledge, we are thus the first to use a recognition task as an indicator of attention to sexual stimuli.

In the first study that used the dot-probe task for sexual pictures, Prause and colleagues (2008) found an unexpected negative relationship between attention bias towards sexual pictures and a broadly defined latent factor of sexual desire. In mixed trials, participants with higher levels of sexual desire were slower to detect probes replacing sexual pictures. Since the effect was rather robust and affected three major domains of sexual desire (desire for autoerotic sexual activity, desire for sexual activity with a partner, and overall propensity for sexual excitation), we concluded it should be manifest in 
172 other domains as well. To explore this further, we employed a revised Sociosexual Orientation

173 Inventory (SOI-R; Penke \& Asendorpf, 2008), because it features a sociosexual desire component

174 which is described as sexual desire targeted at potential mates. Additionally, we used Beck

175 Depressive Inventory (BDI-II; Ptáček, Raboch, Vňuková, Hlinka, \& Anders, 2016) to control for

176 depressive symptoms which could negatively affect the levels of sexual desire in general (Frohlich \&

177 Meston, 2002) and therefore also any attention directed at sexual stimuli.

\section{Materials and Methods}

\section{$179 \quad 2.1 \quad$ Participants}

180 An a priori power analysis was conducted using G*Power 3.1.9.2 (Faul, Erdfelder, Lang, \& Buchner, 181 2007) to test the difference between two dependent group means using a two-tailed test, a medium 182 effect size $d=0.34$ (the effect size estimate for the dot-probe task with sexual stimuli given by 183 Strahler et al., 2019), and an alpha of 0.05. Results showed that a sample of 93 participants was 184 required to achieve a power of 0.90 .

A total of 119 Czech students were recruited at the Charles University campus. Data from six participants (four women, two men) were excluded from analyses due to high error rate (20\% or more during the dot-probe task), leaving 113 participants $\left(M_{\text {age }}=22.00, S D_{\text {age }}=4.47,60.2 \%\right.$ females). This number was substantially higher than the desired sample size. All participants received a remuneration of $100 \mathrm{CZK}$ (app. 4 EUR) for participation. Levels of depressive symptoms in our sample $(M=11.848, S D=9.254)$ were comparable to those of a general healthy Czech population under thirty $(M=10.73, S D=11.53$; Ptáček et al., 2016).

\subsection{Measures}

193 The dot-probe task design was initially partially adapted from Prause, Janssen, and Hetrick (2008)

194 but during pilot testing, most participants found it too easy. To increase the difficulty, we employed a 195 modified version of the dot-probe task in which participants were asked to respond to probes'

196 direction rather than merely their position.

\subsubsection{The modified dot-probe task}

In the dot-probe task, each trial started with an intertrial black screen displayed for 500, 750, 1000, or 1500 milliseconds followed by a fixation cross (1000 ms). Next, laterally randomized sexual and neutral pictures were simultaneously presented on the screen for $500 \mathrm{~ms}$. Then the pictures disappeared and one was replaced by a probe in the form of an arrowhead pointing left or right, which stayed on the screen until a participant responded (see Figure 1). Participants were instructed to press quickly the key assigned for the probe's direction ( $\mathrm{Q}$ for arrow pointing left, $\mathrm{P}$ for arrow pointing right). The lateral position of the probe, which picture category it replaced, and which direction it indicated were all randomized. The dot-probe task started with 20 training trials and continued with two blocks of 50 experimental trials, whereby each block used identical but randomly paired pictures.

\subsubsection{Picture recognition task}

In the picture recognition task, participants were presented with a series of pictures, some of which appeared previously in the dot-probe task (50 sexual, 50 neutral, and 40 training pictures), and some which functioned as distractors (225 pictures). By pressing assigned keys, participants were

212 instructed to indicate whether they saw a picture previously during the dot-probe task (P) or not (Q). 
213 Participants went through the picture recognition task at their own pace because each picture stayed

214 on the screen until a response was produced.

215 All stimuli were presented and responses recorded using E-Prime software package 2.0 (Schneider,

216 Eschman, \& Zuccolotto, 2002).

\section{$217 \quad$ 2.2.3 Questionnaires}

218 All participants completed a short battery of questionnaires consisting of the Screening Questionnaire

219 for psychiatric disorders, BDI-II (Beck, Steer, \& Brown, 1996), SOI-R (Penke \& Asendorpf, 2008),

220

221

222

223 and Emotion Regulation Questionnaire (ERQ; Gross \& John, 2003). Back-translated and standardized Czech version of BDI-II (Ptáček et al., 2016) and back-translated versions of SOI-R and ERQ questionnaires were used. ERQ values were collected for different study and will be reported elsewhere.

\subsection{Materials}

225

226

227

228

All pictures used in the study were taken from standardized datasets, namely IAPS (Lang, Bradley, \& Cuthbert, 1997) and NAPS (Marchewka, Zurawski, Jednoróg, \& Grabowska, 2014). A total of 365 pictures depicting male-female couples, males, and females were pre-rated by 289 online raters for the sexual/nonsexual content. On this basis, we selected 57 sexual (IAPS: 4604, 4608, 4647, 4649, 4651, 4652, 4656, 4658, 659, 4660, 4664, 4666, 4668, 4669, 4670, 4672, 4676, 4677, 4680, 4681, 4683, 4687, 4690, 4692, 4693, 4694, 4695, 4697, 4698, 4800, 4810, 4232, 4290, 4311, 4460, 4470, 4490, 4550; NAPS: Opposite-sex_couple_002_h,Opposite-sex_couple_005_h, Oppositesex_couple_008_h, Opposite-sex_couple_009_h, Opposite-sex_couple_012_h,Oppositesex_couple_014_h, Opposite-sex_couple_017_v, Opposite-sex_couple_018_h, Oppositesex_couple_023_h, Opposite-sex_couple_024_h,Opposite-sex_couple_025_h, Oppositesex_couple_033_v, Female_002_h, Female_003_v, Female_018_h, Female_020_v, Male_008_v, Male_014_v, Male_022_v) and 97 neutral (IAPS: 2389, 2390, 4598, 4600, 4601, 4603, 4605, 4610, 4612, 4616, 4617, 4619, 4626, 4628, 4641, 4700, 8032, 8033, 8461, 8470, 2394, 2010, 2020, 2101, 2190, 2221, 2230, 2393, 2484, 2485, 2488, 2489, 2493, 2630, 5410, 7550, 8080, 8130, 8160, 8320, 8350, 9160; NAPS: Opposite-sex_couple_006_h,Opposite-sex_couple_019_v, Oppositesex_couple_021_h, Opposite-sex_couple_026_h,Opposite-sex_couple_028_h, Oppositesex_couple_032_h, Opposite-sex_couple_035_h, Opposite-sex_couple_037_h, Oppositesex_couple_038_h, Opposite-sex_couple_039_h,Opposite-sex_couple_041_v, Oppositesex_couple_042_h, Opposite-sex_couple_044_h, Faces_356_h, Faces_162_h, Faces_242_h, Faces_278_h, Faces_301_h, Faces_307_v, Faces_318_v, Faces_327_v, Faces_335_h, Faces_346_v, Faces_342_h, Faces_281_h, Faces_288_h, Faces_305_h, Faces_354_v, People_162_h, Faces_246_v, Faces_249_v, Faces_314_h, Faces_316_h, Faces_325_v, Faces_337_h, Faces_348_h, Faces_144_h, Faces_154_h, Faces_158_h, Faces_167_v, Faces_185_v, Faces_187_v, Faces_189_v, Faces_191_v, Faces_192_h, Faces_196_h, Faces_204_v, Faces_211_h, Faces_226_h, Faces_238_h, Faces_251_v, Faces_258_h, Faces_312_h, Faces_326_h, Faces_332_v) pictures. 43 sexual pictures depicted naked heterosexual pairs engaging in sexual activities, while 14 sexual pictures depicted nudity ( 7 showed a naked man and 7 a naked woman). These latter pictures were used in the female and male version of the task, respectively. For each of the 50 sexual pictures, there was a neutral picture matched for content, i.e. the neutral pictures depicted mainly clothed heterosexual pairs, single women or single men. Another 40 more neutral pictures depicting clothed men (one man per picture) were used for the training phase of the dot-probe task. The rest of the 365 original pictures was used as distractors for the picture recognition task. 


\subsection{Procedure}

258 Students were addressed by an assistant at the university campus and offered participation in the study. Upon agreeing to participate, they received further information to read, their eventual questions were answered, and all participants signed the informed consent form. At the outset, each participant completed the Screening Questionnaire, BDI-II, SOI-R, and ERQ. Next, each participant was seated in front of a personal computer and the dot-probe task and PRT were administered. Finally, each participant was debriefed and paid before leaving. The whole procedure took app. 30 minutes.

\subsection{Data analysis}

Data analyses were performed using R (R. C. Team, 2019) and JASP (J. Team, 2019). Dot-probe task reaction times $(\mathrm{RT})$ were trimmed by incorrect responses $(0.67 \%$ of all data) and values $4 S D$ above and below group mean (2.65\% of all data). Descriptive statistics are presented in Table 1 . Due to technical issues, data were lost from 8 participants for BDI-II and 11 participants for SOI-R.

Attention bias index was computed for each participant by subtracting the mean reaction time in mixed pair trials where probe replaced a sexual picture from the mean reaction time in mixed pair trials where probe replaced a neutral picture. Positive values indicate vigilance to sexual pictures, whereas negative values indicate avoidance/difficulty to disengage from sexual pictures.

274 Because of normality violations $(p<0.001)$, a two-tailed Wilcoxon signed-rank test was used to 275 calculate differences in reaction times between trials where probe replaced a neutral picture and trials where the probe replaced a sexual picture. Further, two-tailed Mann-Whitney U test was used to calculate the differences in attention bias index between men and women.

The relationship between attention bias index and sum scores of the questionnaires was tested using

279 Spearman's rank correlation coefficient.

280 Because of concerns regarding low reliability of the dot-probe paradigm (Schmukle, 2005; trials with the second half.

We calculated accuracy scores (\% of correct recognitions) for the picture recognition test. Correct recognition meant marking sexual, neutral, and training pictures as previously seen and marking distractors as not seen before. Since distractor accuracy scores were high $(M=87.8 \%, S D=12.2$ $\%)$, they were screened for outliers and not used in the final analysis. The rest of accuracy scores was included in a mixed ANOVA with one within-subject factor (sexual, neutral, training) and one between-subjects factor (men, women).

289 For all tests, the alpha level of statistical significance was set to 0.05 and effect sizes and confidence 290 intervals calculated.

\subsection{Ethics statement}

292 Participants were informed about the whole procedure and signed written informed consent. The y 


\subsection{Modified dot-probe task}

298

299

300

301

302

303

304

305

306

307

308

309

310

311

312

313

314

315

316

317

318

319

320

321

322

323

324

325

326

327

328

329

330

331

332

333

334

335

A Wilcoxon's signed-rank test showed that the difference between trials with probe replacing a sexual picture $(M d n=611.06 \mathrm{~ms})$ and trials with probe replacing a neutral picture $(M d n=615.41$ $\mathrm{ms})$ was statistically not significant $(\mathrm{W}=3868, p=0.064$, Hodges-Lehmann estimate $=4.520 \mathrm{~ms}$, $95 \% C I[-0.247 ; 9.836]$, matched rank biserial correlation $\left.r_{B}=0.201,95 \% C I[-0.009 ; 0.394]\right)$. Moreover, Mann-Whitney U test showed that the difference in attention bias index between men $(M d n=2.495 \mathrm{~ms})$ and women $(M d n=2.340 \mathrm{~ms})$ was not statistically significant either $(\mathrm{W}=1448, p$ $=0.633$, Hodges - Lehmann estimate $=-1.083 \mathrm{~ms}, 95 \%$ CI $[-6.113 ; 3.659]$, matched rank biserial correlation $\left.r_{B}=-0.054,95 \% C I[-0.266 ; 0.163]\right)$. See Figure 2 for illustration and Table 1 for summary.

All reliability estimates were well above 0.80 (see Table 2) and statistically significant (all $p<$ 0.001).

\subsection{Attention bias and questionnaires}

We found positive correlations between attention bias index and the SOI-R Attitude subscale $\left(r_{s}(100)\right.$ $=0.274,95 \% C I[0.084 ; 0.445])$ and between attention index bias index and SOI-R Desire subscale (Desire subscale: $\left.r_{s}(100)=0.232,95 \% C I[0.040 ; 0.407]\right)$, which were statistically significant $(p=$ 0.005 and 0.019 , respectively). There was no statistically significant correlation between attention bias index and either the SOI-R Behavior subscale $\left(r_{s}(100)=0.147,95 \% C I[-0.048 ; 0.331], p=\right.$ $0.139)$ or the BDI-II sum score $\left(r_{s}(103)=0.078,95 \% C I[-0.116 ; 0.266], p=0.430\right)$.

\subsection{Picture recognition task}

Mauchly's test indicated that the assumption of sphericity for the ANOVA had been violated $\left(\chi^{2}(2)=\right.$ $0.551, p<0.001)$, which is why degrees of freedom were corrected using Greenhouse-Geisser estimates of sphericity $(\varepsilon=0.696)$. There was a main effect of the within-subject factor $(\mathrm{F}(1.380$, $\left.153.222)=213, p<0.001, \eta^{2}=0.427\right)$. Post hoc tests using Bonferroni correction revealed that sexual pictures $(M=48.4 \%, S D=21.1)$ were recognized more often than either the neutral $(M=$ $19.3 \%, S D=14.3, d=1.461,95 \% C I[1.193 ; 1.724], p<0.001)$ or the training pictures $(M=15.4$ $\%, S D=15.0, d=1.445,95 \% C I[1.180 ; 1.708], p<0.001)$. Moreover, there was a significant difference between the two latter conditions $(d=0.353,95 \% C I$ [0.162;0.542], $p<0.001)$. Neither the main effect of the between-subjects factor $\left(\mathrm{F}(1,111)=0.521, \eta^{2}=0.005\right)$ or interaction between the two factors $\left(\mathrm{F}(1.380,153.222)=2.335, \eta^{2}=0.005\right)$ were statistically significant $(p=0.472$ and 0.118 , respectively). See Figure 3 for illustration and Table 3 for a summary.

\section{Discussion}

The aim of present study was to strengthen the evidence for attention bias towards sexual stimuli in a nonclinical population as measured by the dot-probe task. Contrary to all previous research which worked with the dot-probe task paradigm for sexual pictures, our main findings show no evidence of such bias in our data.

What we did find, however, was a large difference in recognition rates for all experimental categories of pictures presented in the picture recognition task. Participants were most successful in recognizing sexual pictures, achieving recognition rate of almost $50 \%$. One might be tempted to explain this as 
336

337

338

339

340

341

342

343

344

345

346

347

348

349

350

351

352

353

354

355

356

357

358

359

360

361

362

363

364

365

366

367

368

369

370

371

372

373

374

375

376

377 random responding, but in the light of markedly lower recognition rates for neutral and training pictures, it is probably not the case. Neutral pictures were successfully recognized at just below a $20 \%$ rate and training pictures at $15 \%$ rate. Since neutral pictures were seen by participants at least twice, once in each block, while training pictures only once at the beginning of the dot-probe task, one might expect such result. The recognition task thus represents a strong, albeit indirect, confirmation of the assumption which the use of the dot-probe task is based on, namely that sexual pictures indeed attract spatial attention.

Our sample did not differ in depressive symptoms from normal population and depressive symptomatology did not affect the attention bias in any way. On the other hand, we found a weak but significant correlation between attention bias, SOI-R Attitude subscale, and the SOI-R Desire subscale. Higher ratings of openness to uncommitted sex and higher sociosexual desire were linked with a faster identification of probes replacing sexual pictures. These results are clearly divergent from Prause and colleagues' (2008) findings of a negative relationship between attention bias and sexual desire. Even so, confidence intervals indicate that the real effect may well be extremely small. We see this inconsistency with previous research as yet another sign of weak reliability of the dotprobe paradigm (see below).

It is but natural that one should ask why the present study found no attention bias towards sexual pictures. There are several possible reasons:

1) Our results may be related to specific variations in the methodology. The $500 \mathrm{~ms}$ presentation time may have given participants enough time to freely shift attention before the probe appeared (Cooper \& Langton, 2006; Jiang \& Vartanian, 2018), the stimuli may have been too weak to capture attention, or they may have been too complex (Miller \& Fillmore, 2010). It is also possible that the more demanding probe-identifying task diverted participants' attention, etc. The main problem with these explanations is that if small methodology changes weakened the effect as significantly as our results seem to suggest, the effect itself may not be as universal and robust as theorized.

2) The study may have insufficient power to reliably find the effect. If that is the case, given that with a sample size of 113 the study had a $95 \%$ probability of finding the reported effect had it been of size of at least 0.34 , the real effect would have to be much smaller, possibly on the lower bound of reported confidence interval (Strahler et al., 2019).

3) Our findings may represent one of the not so rare cases of false negative results.

4) Attention bias may exist but the effect was obscured by another mechanism which interferes with the way dot-probe task works. It has been suggested (Brauer et al., 2012; Prause et al., 2008) that delayed disengagement, as described in other emotionally arousing stimuli, may turn out to be such a mechanism. A large body of evidence showing a slowdown of reaction times in the presence of sexual stimuli (sexual content-induced delay; for a review, see Imhoff et al., 2019) certainly points in that direction. If that is the case, the dot-probe task is not an appropriate measure for attention bias.

5) The dot-probe task may be a poor measurement instrument. Although we found our results robust and reliable as indicated by the split-half reliability estimate, the dot-probe task measure as such has been criticized for extremely weak reliability (Schmukle, 2005; Staugaard, 2009). It has also been claimed that the dot-probe task cannot effectively distinguish between attention bias and the sexual content-induced delay and struggles to establish reliable findings in other research areas (see Kruijt, Parsons, \& Fox, 2019; Strahler et al., 2019). 
6) The most obvious explanation is that there is no attention bias towards sexual pictures - at least not in a form that can be measured by the dot-probe task - or that it is exceedingly small and easily drowned in a measurement error. Even the evidence from the recognition task is somewhat mixed. Sexual stimuli seem to be prioritized in memory, as shown in high free recall rates (Bradley, Karlsson, \& Lang, 2017; Bush \& Geer, 2001; Pottage \& Schaefer, 2012), and a similar effect might as well apply to recognition of sexual pictures. In this context, the $50 \%$ recognition rate for sexual pictures could be maintained had participants attended to sexual stimuli only half the time and not most of it as predicted. Nonetheless, to the best of our knowledge, this area has not been explored yet. Other sources of evidence in favor of existence of attention bias are not very convincing either. Eye-tracking studies which show early attentional orientation toward sexual pictures (Fromberger et al., 2012; Lykins, Meana, \& Kambe, 2006) are seriously underpowered. The constantly increasing use of erotica in advertisement (Reichert \& Carpenter, 2003; Reichert et al., 1999) may be explained by other properties of sexual stimuli, namely its arousing nature and its effect on memory mentioned just above. To date, there is no convincing evidence for visuospatial attention capture by sexual stimuli (Imhoff et al., 2019; Strahler et al., 2019).

\section{Conclusion}

The present study employed a modified dot-probe task to measure attention bias toward sexual pictures in a non-clinical population. Additionally, we used a simple picture recognition task as a measure of dot-probe task validity. Although the findings of the picture recognition task did suggest increased attention towards sexual pictures, dot-probe task results showed no effect whatsoever. Moreover, there were no differences between men and women. We discussed several implications and possible explanations of our non-significant finding. In conclusion, our findings and literature search suggest that either the attention bias toward sexual pictures is rather small, the bias in the theorized form does not exist, or the dot-probe task is not a reliable tool to assess it.

\section{Conflict of Interest}

403 The authors declare that the research was conducted in the absence of any commercial or financial

404 relationships that could be construed as a potential conflict of interest.

\section{$405 \quad 7 \quad$ Funding}

406 The study was supported by the project GACR (GA17-11004S) and by the project NPU I (LO1611).

\section{Acknowledgments}

408 We would like to thank the Faculty of Humanities and the Faculty of Science of the Charles

409 University for letting us use their premises for data collection and AP for English proofreading.

\section{References}

411 Aspen, V., Darcy, A. M., \& Lock, J. (2013). A Review of Attention Biases in Women with Eating

412 Disorders. Cognition and Emotion, 27(5), 820-838. https://doi.org/10.1080/02699931.2012.749777

413 Barlow, D. H. (1986). Causes of Sexual Dysfunction. The Role of Anxiety and Cognitive

414 Interference. Journal of Consulting and Clinical Psychology, 54(2), 140-148.

415 https://doi.org/10.1037/0022-006X.54.2.140 
416 Beck, A. T., Steer, R. A., \& Brown, G. K. (1996). Beck depression inventory-II. San Antonio, TX:

417 The Psychological Corporation.

418 Bradley, B. P., Mogg, K., Falla, S. J., \& Hamilton, L. R. (1998). Attentional Bias for Threatening 419 Facial Expressions in Anxiety: Manipulation of Stimulus Duration. Cognition and Emotion, 12(6), 420 737-753. https://doi.org/10.1080/026999398379411

421 Bradley, M. M., Karlsson, M., \& Lang, P. J. (2017). Assessing Hedonic Bias in Emotional Scene 422 Memory: Implications for Clinical Research. Zeitschrift Für Psychologie / Journal of Psychology, 423 225(3), 223-231. https://doi.org/10.1027/2151-2604/a000306

424 Brauer, M., Van Leeuwen, M., Janssen, E., Newhouse, S. K., Heiman, J. R., \& Laan, E. (2012). 425 Attentional and Affective Processing of Sexual Stimuli in Women with Hypoactive Sexual Desire 426 Disorder. Archives of Sexual Behavior, 41(4), 891-905. https://doi.org/10.1007/s10508-011-9820-7

427 Bush, S. I., \& Geer, J. H. (2001). Implicit and Explicit Memory of Neutral, Negative Emotional, and 428 Sexual Information. Archives of Sexual Behavior, 30(6), 615-631. https://doi.org/10.1023/A:1011915001416

430 Cooper, R. M., \& Langton, S. R. H. (2006). Attentional Bias to Angry Faces Using the Dot-Probe 431 Task? It Depends When You Look for It. Behaviour Research and Therapy, 44(9), 1321-1329. 432 https://doi.org/10.1016/j.brat.2005.10.004

433 de Jong, D. C. (2009). The Role of Attention in Sexual Arousal: Implications for Treatment of Sexual 434 Dysfunction. Journal of Sex Research, 46(2-3), 237-248.

435 https://doi.org/10.1080/00224490902747230

436 Dehghani, M., Sharpe, L., \& Nicholas, M. K. (2003). Selective Attention to Pain-Related Information 437 438 in Chronic Musculoskeletal Pain Patients. Pain, 105(1-2), 37-46. https://doi.org/10.1016/S03043959(03)00224-0

Doornwaard, S. M., van Den Eijnden, R. J. J. M., Johnson, A., \& ter Bogt, T. F. M. (2014). Exposure to Sexualized Media Content and Selective Attention for Sexual Cues: An Experimental Study. Computers in Human Behavior, 41, 357-364. https://doi.org/10.1016/j.chb.2014.10.037

Faul, F., Erdfelder, E., Lang, A.-G., \& Buchner, A. (2007). G*Power 3: A Flexible Statistical Power Analysis Program for the Social, Behavioral, and Biomedical Sciences. Behavior Research Methods, 39(2), 175-191.

448 Fromberger, P., Jordan, K., von Herder, J., Steinkrauss, H., Nemetschek, R., Stolpmann, G., \& 449 Müller, J. L. (2012). Initial Orienting Towards Sexually Relevant Stimuli: Preliminary Evidence 450 from Eye Movement Measures. Archives of Sexual Behavior, 41(4), 919-928.

451 https://doi.org/10.1007/s10508-011-9816-3 
452 Georgiou, G. A., Bleakley, C., Hayward, J., Russo, R., Dutton, K., Eltiti, S., \& Fox, E. (2005).

453 Focusing on Fear-Attentional Disengagement from Emotional Faces. Visual Cognition, 12(1), 145454 158. https://doi.org/10.1080/13506280444000076.Focusing

455 Gross, J. J., \& John, O. P. (2003). Individual Differences in Two Emotion Regulation Processes:

456 Implications for Affect, Relationships, and Well-Being. Journal of Personality and Social

457 Psychology, 85(2), 348-362. https://doi.org/10.1037/0022-3514.85.2.348

458 Hennink-Kaminski, H. J., \& Reichert, T. (2011). Using Sexual Appeals in Advertising to Sell

459 Cosmetic Surgery: A Content Analysis from 1986 to 2007. Sexuality and Culture, 15(1), 41-55.

460 https://doi.org/10.1007/s12119-010-9081-y

461 Hogarth, L. C., Mogg, K., Bradley, B. P., Duka, T., \& Dickinson, A. (2003). Attentional Orienting

462 Towards Smoking-Related Stimuli. Behavioural Pharmacology, 14(2), 153-160.

463 https://doi.org/10.1097/00008877-200303000-00007

464 Imhoff, R., Barker, P., \& Schmidt, A. F. (2019). To What Extent Do Erotic Images Elicit

465 Visuospatial versus Cognitive Attentional Processes? Consistent Support for a (Non-Spatial) Sexual

466 Content-Induced Delay. Archives of Sexual Behavior, 1-20. https://doi.org/10.1007/s10508-019-

$467 \quad 01512-0$

468

Ioannidis, J. P. A., Munafò, M. R., Fusar-Poli, P., Nosek, B. A., \& David, S. P. (2008). Publication

469 and Other Reporting Biases in Cognitive Sciences: Detection, Prevalence and Prevention. Trends in

470 Cognitive Sciences, 18(5), 235-241. https://doi.org/10.1038/jid.2014.371

471

Jiang, M. Y. W., \& Vartanian, L. R. (2018). A Review of Existing Measures of Attentional Biases in

472

473 Body Image and Eating Disorders Research. Australian Journal of Psychology, 70(1), 3-17. https://doi.org/10.1111/ajpy.12161

Kagerer, S., Wehrum, S., Klucken, T., Walter, B., Vaitl, D., \& Stark, R. (2014). Sex Attracts: Investigating Individual Differences in Attentional Bias to Sexual Stimuli. PLoS ONE, 9(9). https://doi.org/10.1371/journal.pone.0107795

477 Kruijt, A.-W., Parsons, S., \& Fox, E. (2019). A Meta-Analysis of Bias at Baseline in RCTs of Attention Bias Modification: No Evidence for Dot-Probe Bias Towards Threat in Clinical Anxiety and PTSD. Journal of Abnormal Psychology, 128(6), 563-573.

480 Lang, P. J., Bradley, M. M., \& Cuthbert, B. N. (1997). International Affective Picture System (IAPS): Technical Manual and Affective Ratings. NIMH Center for the Study of Emotion and Attention. Retrieved from http://www.unifesp.br/dpsicobio/adap/instructions.pdf 3(4), 525-551. https://doi.org/10.1016/0010-0285(72)90021-7 and Non-Erotic Stimuli Using Eye-Tracking Methodology. Archives of Sexual Behavior, 35(5), 569575. https://doi.org/10.1007/s10508-006-9065-Z 
490

491

492

493

494

495

496

497

498

499

500

501

502

503

504

505

506

507

508

509

510

511

512

513

514

515

516

517

518

519

520

521

522

523

524

525

526
Marchewka, A., Zurawski, L., Jednoróg, K., \& Grabowska, A. (2014). The Nencki Affective Picture System (NAPS): Introduction to a Novel, Standardized, Wide-Range, High-Quality, Realistic Picture Database. Behavior Research Methods, 46(2), 596-610. https://doi.org/10.3758/s13428-013-0379-1

Mechelmans, D. J., Irvine, M., Banca, P., Porter, L., Mitchell, S., Mole, T. B., ... Voon, V. (2014). Enhanced Attentional Bias Towards Sexually Explicit Cues in Individuals with and Without Compulsive Sexual Behaviours. PLoS ONE, 9(8). https://doi.org/10.1371/journal.pone.0105476

Miller, M. A., \& Fillmore, M. T. (2010). The Effect of Image Complexity on Attentional Bias Toward Alcohol-Related Images in Adult Drinkers. Addiction, 105(5), 883-890.

https://doi.org/10.1038/jid.2014.371

Mogg, K., Philippot, P., \& Bradley, B. P. (2004). Selective Attention to Angry Faces in Clinical Social Phobia. Journal of Abnormal Psychology, 113(1), 160-165. https://doi.org/10.1037/0021843X.113.1.160

Navon, D., \& Margalit, B. (1983). Allocation of Attention According to Informativeness in Visual Recognition. The Quarterly Journal of Experimental Psychology, 35(3), 497-512.

https://doi.org/10.1080/14640748308402484

Penke, L., \& Asendorpf, J. B. (2008). Beyond Global Sociosexual Orientations: A More Differentiated Look at Sociosexuality and Its Effects on Courtship and Romantic Relationships. Journal of Personality and Social Psychology, 95(5), 1113-1135. https://doi.org/10.1037/00223514.95.5.1113

Posner, M. I., \& Cohen, Y. (1984). Components of Visual Orienting. In H. Bouma \& D. Bouwhuis (Eds.), Attention and Performance (pp. 531-556). London: Erlbaum. https://doi.org/10.2307/414713

Posner, M. I., Snyder, C. R., \& Davidson, B. J. (1980). Attention and the Detection of Signals. Journal of Experimental Psychology: General, 109(2), 160-174. https://doi.org/10.1037/00963445.109.2.160

Pottage, C. L., \& Schaefer, A. (2012). Visual Attention and Emotional Memory: Recall of Aversive Pictures is Partially Mediated by Concurrent Task Performance. Emotion, 12(1), 33-38. https://doi.org/10.1037/a0024574

Prause, N., Janssen, E., \& Hetrick, W. P. (2008). Attention and Emotional Responses to Sexual Stimuli and Their Relationship to Sexual Desire. Archives of Sexual Behavior, 37(6), 934-949. https://doi.org/10.1007/s10508-007-9236-6

Ptáček, R., Raboch, J., Vňuková, M., Hlinka, J., \& Anders, M. (2016). Beckova Škála Deprese BDIII - Standardizace a Využití v Praxi. Česká a Slovenská Psychiatrie, 112(6), 270-274.

Reichert, T., \& Carpenter, C. (2003). An Update on Sex in Magazine Advertising: 1983 to 2003. Journalism \& Mass Communication Quarterly, 81(4), 823-837.

Reichert, T., Lambiase, J., Morgan, S., Carstarphen, M., \& Zavoina, S. (1999). Cheesecake and Beefcake: No Matter How You Slice It, Sexual Explicitness in Advertising Continues to Increase. Journalism \& Mass Communication Quarterly, 76(1), 7-20. 
Rinck, M., Becker, E. S., Kellermann, J., \& Roth, W. T. (2003). Selective Attention in Anxiety: Distraction and Enhancement in Visual Search. Depression and Anxiety, 18(1), 18-28. https://doi.org/10.1002/da.10105

Schmukle, S. C. (2005). Unreliability of the Dot Probe Task. European Journal of Personality, 19(7), 595-605. https://doi.org/10.1002/per.554

Schneider, W., Eschman, A., \& Zuccolotto, A. (2002). E-Prime (Version 2.0). Pittsburgh, PA: Psychology Software Tools Inc.

Seehuus, M. (2015). Discrepant Attentional Biases Toward Sexual Stimuli. University of Vermont. Retrieved from https://scholarworks.uvm.edu/graddis/416

Snowden, R. J., Curl, C., Jobbins, K., Lavington, C., \& Gray, N. S. (2016). Automatic Direction of Spatial Attention to Male Versus Female Stimuli: A Comparison of Heterosexual Men and Women. Archives of Sexual Behavior, 45(4), 843-853. https://doi.org/10.1007/s10508-015-0678-y

Spiering, M., \& Everaerd, W. (2007). The Sexual Unconscious. In E. Janssen (Ed.), The Psychophysiology of Sex (pp. 166-184). Indiana University Press.

Staugaard, S. R. (2009). Reliability of Two Versions of the Dot-Probe Task Using Photographic Faces. Psychology Science Quarterly, 51(3), 339-350.

Strahler, J., Baranowski, A. M., Walter, B., Huebner, N., \& Stark, R. (2019). Attentional Bias Toward and Distractibility by Sexual Cues: A Meta-Analytic Integration. Neuroscience and Biobehavioral Reviews, 105(June), 276-287. https://doi.org/10.1016/j.neubiorev.2019.07.015

Team, J. (2019). JASP (Version 0.11).

Team, R. C. (2019). R: A Language and Environment for Statistical Computing. Vienna, Austria: R Foundation for Statistical Computing. Retrieved from https://www.r-project.org/

Townshend, J. M., \& Duka, T. (2001). Attentional Bias Associated with Alcohol Cues: Differences Between Heavy and Occasional Social Drinkers. Psychopharmacology, 157(1), 67-74. https://doi.org/10.1007/s002130100764

Wenzel, A., \& Holt, C. S. (1999). Dot Probe Performance in Two Specific Phobias. British Journal of Clinical Psychology, 38(4), 407-410. https://doi.org/10.1348/014466599163006

Wirtz, J. G., Sparks, J. V, \& Zimbres, T. M. (2017). The Effect of Exposure to Sexual Appeals in Advertisements on Memory, Attitude, and Purchase Intention: A Meta-Analytic Review. International Journal of Advertising, 37(2), 168-198. https://doi.org/10.1080/02650487.2017.1334996

\section{Tables}

Table 1. Dot-probe task: Mean RT (ms), median, and SD. 


\begin{tabular}{|c|c|c|c|c|c|c|c|c|c|}
\hline & \multicolumn{3}{|c|}{ All $(\mathrm{N}=113)$} & \multicolumn{3}{|c|}{ Men $(n=45)$} & \multicolumn{3}{|c|}{ Women $(n=68)$} \\
\hline & Mean & Median & SD & Mean & Median & SD & Mean & Median & SD \\
\hline \multirow[t]{2}{*}{ All trials } & 628.24 & 613.95 & 128.92 & 601.96 & 603.76 & 106.15 & 645.62 & 621.59 & 140.05 \\
\hline & 2 & 0 & 6 & 9 & 8 & 3 & 9 & 4 & 6 \\
\hline \multirow[t]{2}{*}{ Trial $_{\text {sex }}$} & 624.91 & 611.05 & 131.81 & 597.88 & 608.26 & 104.69 & 642.79 & 612.55 & 145.01 \\
\hline & 4 & 9 & 0 & 9 & 0 & 2 & 9 & 1 & 6 \\
\hline Trial $_{\text {neutra }}$ & 631.65 & 615.40 & 127.60 & 606.06 & 597.30 & 109.21 & 648.59 & 636.19 & 136.58 \\
\hline 1 & 4 & 8 & 3 & 3 & 6 & 3 & 0 & 0 & 2 \\
\hline $\mathrm{AB}$ & 3.370 & 2.495 & 14.607 & 4.087 & 2.495 & 13.169 & 2.895 & 2.340 & 15.563 \\
\hline
\end{tabular}

Note $:$ Trial $_{\text {sex }}=$ Trials with probe replacing sexual picture, Trial $_{\text {neutral }}=$ Trials with probe replacing neutral picture, $\mathrm{AB}=$ Attention bias index

561 Table 2. Dot-probe task: Split-half reliability estimates.

\begin{tabular}{lllll}
\hline & Split half $r$ & CI 95\% & $\rho_{\text {SP }}$ & $\mathrm{p}$ \\
\hline All trials & 0.894 & {$[0.849 ; 0.926]$} & 0.944 & $<0.001$ \\
Trial $_{\text {sex }}$ & 0.881 & {$[0.831 ; 0.917]$} & 0.937 & $<0.001$ \\
Trial $_{\text {neutral }}$ & 0.881 & {$[0.831,0.917]$} & 0.937 & $<0.001$ \\
\hline
\end{tabular}

Note $:$ Trial $_{\text {sex }}=$ Trials with probe replacing sexual picture, Trial $_{\text {neutral }}=$ Trials with probe replacing neutral picture, $\rho_{\mathrm{SP}}=$ split-half reliability coefficient predicted by Spearman-Brown formula

562 Table 3. Picture recognition task: Mean accuracy scores (\%), Median, and SD.

\begin{tabular}{|c|c|c|c|c|c|c|c|c|c|}
\hline & \multicolumn{3}{|c|}{ All $(\mathrm{N}=113)$} & \multicolumn{3}{|c|}{ Men $(n=45)$} & \multicolumn{3}{|c|}{ Women $(n=68)$} \\
\hline & Mean & Median & SD & Mean & Median & SD & Mean & Median & SD \\
\hline Sexual & 48.389 & 48.0 & 21.091 & 49.867 & 50.0 & 21.786 & 47.412 & 45.0 & 20.724 \\
\hline Neutral & 19.345 & 16.0 & 14.326 & 16.356 & 14.0 & 13.405 & 21.324 & 18.0 & 16.667 \\
\hline Training & 15.376 & 10.0 & 15.049 & 13.556 & 10.0 & 14.157 & 16.581 & 12.5 & 15.597 \\
\hline
\end{tabular}

563

56411 Figure Legends

565 Fig. 1 The modified dot-probe task trial.

566 Fig. 2 Dot-probe task: Reaction times (ms).

567 Fig. 3 Picture recognition task: Correct recognition (\%) for training, neutral, and sexual pictures.

568 Note: $* * * p<0.001$ 